\title{
Impact of low-level laser therapy on the dynamics of pressure ulcer-induced changes considering an infectious agent and cathelicidin LL-37 concentration: a preliminary study
}

\author{
Małgorzata Brauncajs ${ }^{1}$, Krzysztof Ksiąszczyk², Anna Lewandowska-Polak², Katarzyna Gorzela², Janina Grzegorczyk ${ }^{1}$ \\ 1Department of Microbiology and Laboratory Medical Immunology, Medical University of Lodz, Lodz, Poland \\ Internal Medicine Ward, Pabianice Medical Centre, Pabianice, Poland \\ ${ }^{3}$ Department of Immunology, Rheumatology and Allergy, Medical University of Lodz, Lodz, Poland \\ Adv Dermatol Allergol 2018; XXXV (6): 582-586 \\ DOI: https://doi.org/10.5114/ada.2018.77609
}

\begin{abstract}
Introduction: Low-level laser therapy is used in managing chronic wounds including pressure ulcers. Less is known about its impact on the healing process if an inhibitive agent e.g. bacterial infection takes place. Modulating nonspecific immunity processes might eliminate bacteria if laser therapy is applied.

Aim: To investigate the impact of low-level laser therapy on pressure ulcer dynamics considering an infectious agent and cathelicidin LL-37 concentration.

Material and methods: The study comprised 6 patients with pressure ulcers ranging from stage II to III in Torrance classification and 12 patients without pressure ulcers. Venous blood sample and decubitus wound swab were taken - in study groups A at baseline and after 2 weeks; in control group B once - at a specific point of time. The swabs served for species identification. Drug susceptibility of isolated pathogens and cathelicidin LL-37 in serum concentration were measured.

Results: In study group A, the following bacteria predominantly occurred: S. aureus, E. faecalis, P. mirabilis, $P$. aeruginosa, while in control group B, excluding one MRSA case, S. hominis, S. epidermidis, D. nishinomiyaensis, A. haemolyticus (physiological flora) were present. HLGR resistance mechanisms were detected when analyzing drug susceptibility panels. Study group A findings demonstrated a statistically significant difference between the levels of cathelicidin LL-37 concentration at baseline and at the end.

Conclusions: There is insufficient information to accurately determine the effect of LLLT on pressure ulcer dynamics considering an infectious agent. These effects may occur if innate immunity processes are modulated so that laser therapy might eliminate bacteria indirectly.
\end{abstract}

Key words: pressure ulcers, low-level laser therapy.

\section{Introduction}

Pressure ulcers (PU) are hard-to-heal chronic wounds occurring as a result of tissue ischemia and hypoxia as a consequence. The impairment of the healing process occurs, which comes from the destabilization of destruction-reconstruction processes in the new tissue. Pressure ulcers may not only indicate the damage of the skin itself but also lower-located tissues such as subcutaneous, connective or even bone tissue.

Infections are a major problem seriously disturbing an effective treatment of pressure ulcers. They have an inhibitory effect on the pace of the healing process and may deteriorate the general condition of the patient. Pressure ulcers are highly susceptible to the development of bacterial colonization which delays the healing pro- cess [1]. An easy bacterial growth is facilitated by blood circulation impairment in infected tissues. What makes the specific immunity mechanisms ineffective is the fact that usually several types of pathogens develop simultaneously in pressure ulcers. Also, the non-specific immunity mechanisms are impaired within the wound. Given the fact that in the hospital environment a great deal of bacterial strains develop drug resistance to at least several available antibiotics, finding an effective way of healing bacterial infections of pressure ulcers is required.

Besides the common concept of moist wound healing (hydrogel dressings, polyurethane foam, etc.) physiotherapy is a recommended method of supporting pressure ulcer therapy.

\footnotetext{
Address for correspondence: Małgorzata Brauncajs PhD, Department of Microbiology and Laboratory Medical Immunology, Medical University of Lodz, 251 b C5 Pomorska St, 92-213 Lodz, Poland, phone: +48 606262 263, e-mail: malgorzatabrauncajs@gmail.com Received: 5.09.2017, accepted: 3.10.2017.
} 
In physiotherapy, average-power lasers and low-power lasers of 1-500 mW low-level laser therapy (LLLT) are used.

Using a biostimulating laser causes changes at a cellular level. It is explained by absorbing quanta of energy by proteins or organelles which, in turn results in the change of the internal metabolism of the cell. A number of changes that may cause the growth of ATP synthesis, collagen or nucleic acids have been discovered so far.

Laser light also has a considerable impact on the synthesis of different growth factors as well as the regulation of the level of cytokines, which facilitates immune processes and influences the proliferation of cells. Other important effects of laser radiation are the processes leading to enhanced synthesis of endorphins and the decreased conduction of sensory nerve cells, thus causing an analgesic effect.

All the processes make the low-level laser therapy an important therapeutic tool having analgesic, anti-inflammatory and regenerative [2,3] properties. Due to that, lowlevel laser therapy could be applied in managing a variety of chronic wounds including diabetic ulcerations, postsurgical wounds, pressure ulcers and burn wounds [4, 5].

Wherever the imbalance of tissue destruction-reconstruction processes takes place, the impairment of the wound healing process may occur as well. Thus there may be a case of extended or even excessive inflammatory condition within the wound. It significantly extends the phase of healing the wound by inactivation of growth factors and cytokines which, in turn, inhibits wound granulation [6, 7]. Laser therapy used at this stage reduces inflammation by providing optimal conditions required for further effective wound healing.

In the next phase of healing, LLLT may facilitate the proliferation of emerging cells and influence both angiogenesis and the synthesis of growth factors. The processes accelerate wound healing and reduce the risk of its colonization with microorganisms by non-specific immunity stimulation.

Also the effectiveness of human cathelicidin LL-37 seems to confirm its applicability, in terms of wound healing, as it has a considerable impact on the skin immune system as well as by the fact of using PAD (antimicrobial peptides) in drug research to treat ulcerations in 'diabetic foot' or catheter-related infections [8-10].

\section{Aim}

The aim of the research was to estimate the impact of laser therapy on the dynamics of pressure ulcers considering an infectious agent and cathelicidin LL-37 concentration.

\section{Material and methods}

The study comprised 6 patients ( 3 men and 3 women, mean age: $77.3 \pm 7.5$ years, range: $66-88$ years) with pressure ulcers and 12 patients ( 4 men and 8 women, mean age: $43.9 \pm 12.9$ years, range: $24-71$ years) without pressure ulcers, hospitalized in the Internal Medicine Ward. Torrance classification to measure decubitus wounds was used to identify the following:

Study group A - 6 patients with pressure ulcers of stage II-III subjected to laser therapy treatment by means of BTL4000 with laser shower (initial power of the probe at $4 \times$ $400 \mathrm{~mW}$, radiation length at IR $830 \mathrm{~nm}$ ). Each series consisted of 10 laser therapy procedures conducted daily for 5 days a week in accordance with the following parameters: time 1-2 min, surface $20-50 \mathrm{~cm}^{2}$, energy density $2 \mathrm{~J} / \mathrm{cm}^{2}$.

Swabs from the pressure ulcer were taken to a transport tube with Stuart substrate (Medlab Products Sp. z o.o.).

In all the patients, the sample material was collected for laboratory analysis from decubitus wounds of the sick, from the lower leg skin of healthy patients and transported to a test tube with Stuart substrate (Medlab Products Sp. z o.o.). Blood samples were also taken from elbow flexions at a volume of $9 \mathrm{ml}$ per clot from which serum was isolated.

The total number of bacteria cultivable under aerobic conditions was inoculated on Columbia II Agar (Graso BIOTECH) medium with defibrinated $5 \%$ sheep blood. Staphylococci were isolated in the selective Staphylococcus Medium No. 110 (Graso BIOTECH); Gram-negative Enterobacteriaceae in MacConkey (Graso BIOTECH) medium; Enterococci were isolated on a bile-esculin Coccosel Agar (Graso BIOTECH). All the bacteria cultures were incubated at $37^{\circ} \mathrm{C}$ in aerobic conditions for $24 \mathrm{~h}$.

The species identification on the basis of biochemical characteristics of isolated microorganisms and drug susceptibility testing was conducted by means of BD Phoenix (PX 1902) automatic microbiological system. The findings were verified and consistent with EUCAST system expert rules.

ELISA Kit For Cathelicidin Antimicrobial Peptide (CAMP) of USCN Life Science Inc. was used to measure the concentration of cathelicidin LL-37 in serum.

\section{Statistical analysis}

The findings were subjected to statistical analysis by Wilcoxon and U Mann Whitney non-parameter tests by means of Statistica 10 (StatSoft Inc.) software. The materiality level of $p \leq 0.05$ for $\alpha=0.05$ was established as statistically significant.

\section{Results}

One strain of Enterococcus faecalis, one strain of Proteus mirabilis, three strains of Staphylococcus aureus, and two strains of Pseudomonas aeruginosa were isolated in the pre-treatment six-patient group (Figure 1). The results demonstrated the following: Enterococcus faecalis strains, group A, pre-treatment, $100 \%$ resistance to trimethoprim with sulfamethoxazole. HLGR mechanism discovered. 


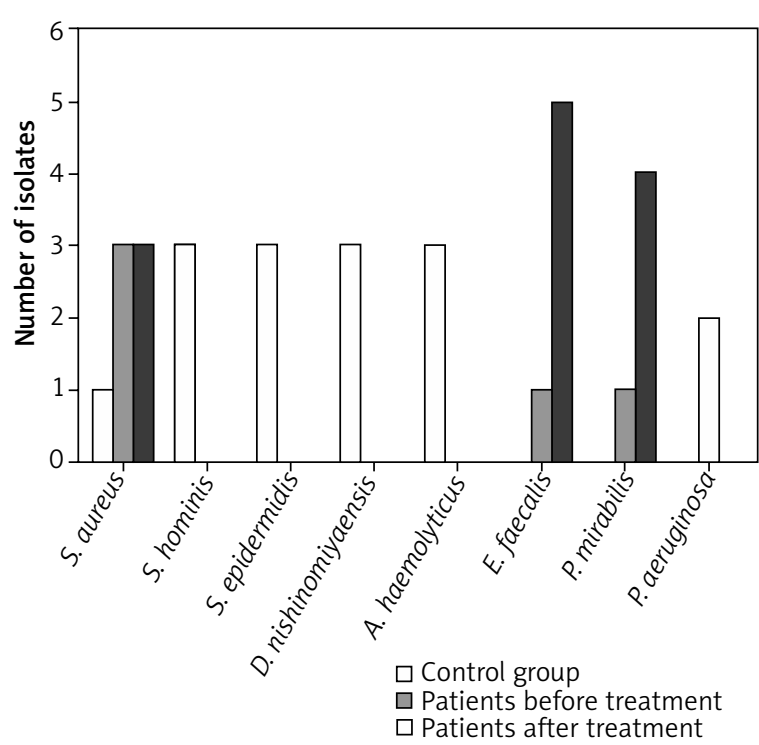

Figure 1. Individual distribution of microbial flora in group $A$ patients, pre- and post-treatment, and in control group B patients

Proteus mirabilis strains $100 \%$ resistant to gentamicin, tobramycin, trimethoprim with sulfamethoxazole as well as levofloxacin and ciprofloxacin.

In Staphylococcus aureus strains, group A, pre-treatment, $66.6 \%$ resistant to ampicillin and penicillin $\mathrm{G}$, $33.3 \%$ resistant to tetracycline.

Fifty percent of Pseudomonas aeruginosa strains resistant to gentamicin, tobramycin, imipenem, nitrofurantoin as well as average resistance to amikacin, meropenem, aztreonam in $50 \%$ of the isolated strains.

In the group of 6 patients, post-treatment, five strains of Enterococcus faecalis, four strains of Proteus mirabilis, three strains of Staphylococcus aureus and one strain of Pseudomonas aeruginosa were isolated (Figure 1).

One hundred percentage of Enterococcus faecalis strains showed resistance to trimethoprim and trimethoprim with sulfamethoxazole, $60 \%$ to nitrofurantoin, and $20 \%$ to synthetic gentamycin in post-treatment, group A. HLGR resistance mechanism identified in 20\% of the strains.

In $25 \%$ of isolated Proteus mirabilis strains, no susceptibility to gentamicin, tobramycin, ampicillin, amoxicillin with clavulanate, trimethoprim, ciprofloxacin, and trimethoprim with sulfamethoxazole was identified. In $25 \%$ of the strain microorganisms, the average susceptibility to imipenem was identified.

In group A, post-treatment, 100\% strains of Staphylococcus aureus were resistant to gentamycin, ampicillin, penicillin G, ciprofloxacin, and moxifloxacin. Resistance to amikacin and tobramycin found in $66.6 \%$, non-susceptibility to trimethoprim in $33.3 \%$, and average susceptibility to amikacin and trimethoprim with sulfamethazole in $33.3 \%$.

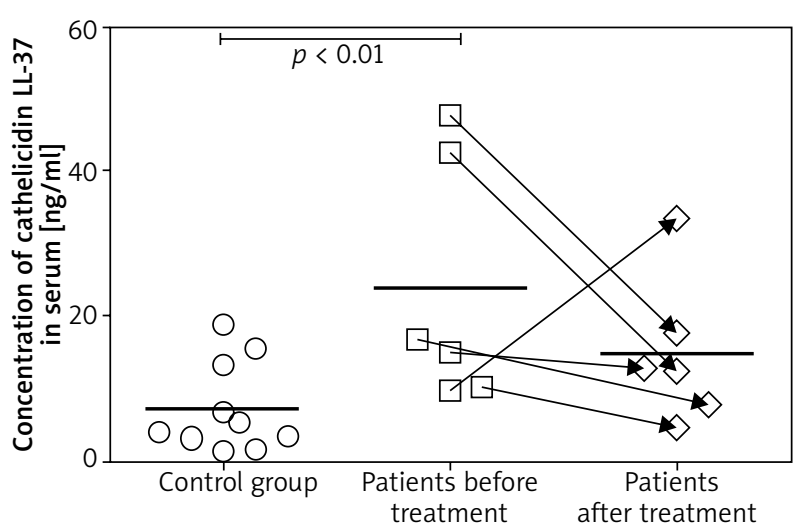

Figure 2. The chart of specific concentrations of cathelicidin LL-37 in group A patients, pre- and post-treatment, including mean value and in control group $B$ patients including mean value

In Pseudomonas aeruginosa, 100\% resistance to gentamicin, tobramycin and average susceptibility to amikacin was found.

In the group of twelve patients without decubitus wounds, one strain of Staphylococcus aureus, three strains of Staphylococcus hominis, three strains of Staphylococcus epidermidis, three strains of Dermacoccus nishinomiyaensis, and three strains of Acinetobacter haemolyticus were isolated (Figure 1). The results demonstrated the following:

In control group B, Staphylococcus aureus 100\% resistant to ampicillin, penicillin G, oxacillin, erythromycin, and phosphomycin. S. aureus (MRSA) isolated here.

Staphylococcus epidermidis 100\% resistant to ampicillin, penicillin $\mathrm{G}$ and erythromycin.

In 100\% of Dermacoccus nishinomiyaensis strains, isolated in control group C, no resistance to antibiotics identified.

One hundred percent of Acinetobacter haemolyticus bacterial isolates, control group B, resistant to aztreonam and nitrofurantoin.

In the group of 6 patients with decubitus wounds subjected to laser therapy, the level of cathelicidin LL-37 concentration was established for each patient before and after the treatment.

In group $\mathrm{A}$, pre-treatment concentration of cathelicidin LL-37 in blood serum at ap. $23.77 \pm 6.90 \mathrm{ng} / \mathrm{ml}$. The lowest cathelicidin LL-37 concentration in sick patients subjected to laser therapy at $80 \mathrm{ng} / \mathrm{ml}$, pre-treatment, and $47.86 \mathrm{ng} / \mathrm{ml}$ at the highest value.

In group A patients subjected to laser therapy, posttreatment value of cathelicidin LL-37 concentration in blood serum at ap. $14.83 \pm 4.16 \mathrm{ng} / \mathrm{ml}$. Its lowest posttreatment concentration was $4.56 \mathrm{ng} / \mathrm{ml}$; the highest value at $33.48 \mathrm{ng} / \mathrm{ml}$ (Figure 2). 
In the twelve-patient control group without decubitus wounds, cathelicidin LL-37 concentration was measured at a reference point of time. In blood serum it was ap. $7.77 \pm 1.97 \mathrm{ng} / \mathrm{ml}$. The lowest concentration at $1.29 \mathrm{ng} / \mathrm{ml}$, the highest value $-18.81 \mathrm{ng} / \mathrm{ml}$ (Figure 2).

The pre-treatment mean value of cathelicidin LL-37 in patients subjected to laser therapy (group A) was 23.77 $\pm 6.90 \mathrm{ng} / \mathrm{ml}$, which makes $305.97 \%$ of the mean value for the control group.

The mean value of cathelicidin LL-37 in the control group was $7.77 \mathrm{ng} / \mathrm{ml}$.

The study group, post-treatment, presented a lower value of cathelicidin LL-37 concentration as compared to baseline value. The mean value of cathelicidin LL-37 concentration decreased by $8.94 \mathrm{ng} / \mathrm{ml}$, which is $37.6 \%$ of the baseline mean value. The pre-treatment and posttreatment difference in cathelicidin LL-37 concentration in group A was statistically significant $(p=0.043)$ only if the patient for whom the decreased cathelicidin concentration level was recorded is ignored in the measurement.

The highest cathelicidin LL-37 post-treatment value recorded in the group of patients subjected to laser therapy (A) at $14.83 \pm 4.16 \mathrm{ng} / \mathrm{ml}$, which makes $190.93 \%$ of the mean value for the control group.

In patients subjected to laser therapy (group A), 5 cases in which cathelicidin LL-37 concentration decreased after treatment were identified. In one case of group A, cathelicidin LL-37 concentration increased after treatment.

\section{Discussion}

Based on the research, the impact of laser therapy on particular species of pathogens cannot be determined due to the heterogeneity of microbial flora colonizing the decubitus wounds in the examined cases. In majority of patients, pre-treatment and post-treatment, more than one strain of bacteria was identified for each patient. $S$. aureus, $P$. mirabilis, E. faecalis, and $P$. aeruginosa were the most commonly identified bacterial strains. Among the strains mentioned above, HLGR (antibiotic) resistance mechanism was discovered (patient 1 and patient 3 of group A). In control group B, except for one case of MRSA strain, bacteria such as S. hominis, S. epidermidis, $D$. nishinomiyaensis, A. haemolyticus, part of physiological flora living on human skin, were found. The situation does not allow to identify a dominant pathogen. Thus, the research results cannot be applied for particular pathogens.

Despite the therapy, in majority of cases the wound was colonized by pathogens other than those observed at the beginning of the study. The probable reason for such a case might be the fact of contaminating the wound with microorganisms living on hospital objects, e.g. beds, bed linen, and clothes directly exposed to human skin.

The similarity of drug susceptibility profiles of the pathogens isolated in certain patients (patients 1-4 of study group A) might suggest an occurrence of nosocomial infection, given the fact that the following factors occurred: serious clinical condition, long hospitalization period, invasive procedures, and chronic diseases predisposing to this type of infection.

In study group A, in which laser therapy was applied, a lower value of cathelicidin LL-37 concentration was observed in five out of 6 patients. In one patient, cathelicidin LL-37 concentration increased in comparison with the baseline value. The decrease in concentration of peptide antibiotics mentioned above, in the group of patients exposed to the low-level laser therapy, might be explained in two respective ways. On the one hand, it may be seen as an effect of anti-inflammatory properties of low-level lasers. Study results, confirmed by other researchers, indicate a decreased concentration of a great number of pro-inflammatory cytokines such as TNF- $\alpha$ or IFN- $\gamma$ [11] when exposed to LLLT. As a hypothesis we may assume that this form of therapy has an impact on the decrease of peptide antibiotics concentration in the group of substances such as cathelicidin LL-37. This assumption, however, requires investigating an impact of laser therapy on other non-specific immunity mechanisms.

In one group A patient, an increased concentration of cathelicidin LL-37 was recorded in comparison with the value of the first reference point. Having been examined towards coexistent diseases, the patient was diagnosed with diabetes. Due to its impact on blood vessels and wound healing properties, the disease might have impaired the regulation mechanisms of cathelicidin LL-37 synthesis [12] in the patient.

The results could not be applied to any other studies as no other publications on the impact of low-level laser therapy on cathelicidin LL-37 concentration, the processes of stimulating non-specific immunity, or supporting the elimination of bacteria had been recorded.

\section{Conclusions}

Multi-resistant (MRSA) bacterial strains [13] are becoming increasingly problematic in the therapy of healing wounds. The infection of the wound extends and impairs the healing process. What seems to be crucial is successful elimination of the pathogen and reactivating normal processes of tissue reconstruction. The situation in which majority of available antibiotics appear to be ineffective in the combat against microorganisms, requires alternative ways of elimination or prevention of infections. Supporting the natural non-specific immunity mechanisms may be an alternative when combating multi-resistant bacteria. 
Low-level laser therapy has potential benefits in the combat against pathogens infecting pressure ulcers. It may be perceived, however, as a factor affecting the modulation of non-specific immunity processes [14, 15] rather than a method of direct elimination of bacteria. In the light of the present scientific opinion it is impossible to identify the characteristics and effectiveness of the reactions. Further studies will be required to answer the enquiries about direct or indirect impact of laser therapy on non-specific response mechanisms as well as whether this activity considerably contributes to more effective elimination of bacteria.

\section{Acknowledgments}

This study was supported by the Medical University of Lodz, Poland (no. 503/1-137-02/503-11-001).

\section{Conflict of interest}

The authors declare no conflict of interest.

\section{References}

1. Atkinson A, Campbell K, Dolynchuk K, et al. Best practices for the prevention and treatment of pressure ulcers. Ostomy Wound Manage 2000; 46: 38-52.

2. Hirasawa T, Ishihara M, Kushibiki T, et al. Regulation of miRNA expression by low-level laser therapy (LLLT) and photodynamic therapy (PDT). Int J Mol Sci 2013; 14: 13542-58.

3. Basso FG, Hebling J, Kurachi C, et al. Biostimulatory effect of low-level laser therapy on keratinocytes in vitro. Lasers Med Sci 2013; 28: 367-74.

4. Bayat M, Ezzati A, Mohsenifar Z, et al. Low-level laser therapy with pulsed infrared laser accelerates third-degree burn healing process in rats. J Rehabil Res Develop 2009; 46: 543-54.

5. Houreld NN, Nteleki B. The use of phototherapy in the treatment of diabetic ulcers. J Endocrinol Metabol Diabetes South Africa 2012; 17: 128-32.

6. Stadelmann WK, Digenis AG, Tobin GR. Physiology and healing dynamics of chronic cutaneous wounds. Am J Surg 1998; 176 Suppl. 2 A: 26-38.

7. Szymczyszyn A, Doroszko A, Szahidewicz-Krupska E, et al. Effects of transdermal low-level laser therapy on endothelial function. Lasers Med Sci 2016; 31: 1301-7.

8. Chromek M. The antimicrobial peptide cathelicidin protects the urinary tract against invasive bacterial infection. Nat Med 2006; 12: 636-41

9. Zasloff M. Antimicrobial peptides of multicellular organisms. Nature 2002; 415: 389-95.

10. Ganz T. Defensins: antimicrobial peptides of innate immunity. Nat Rev Immunol 2003; 3: 710-20.

11. Fukuda TY, Sato MN, Silva SR, et al. Infrared low-level diode laser on inflammatory process modulation in mice: pro- and anti-inflammatory cytokines. Lasers Med Sci 2013; 28: 1305-13.

12. Zanetti M. Cathelicidins, multifunctional peptides of the innate immunity. J Leukoc Biol 2004; 75: 39-48.

13. Thai TP, Houghton PE, Campbell KE, et al. Ultraviolet light $C$ in the treatment of chronic wounds with MRSA: a case study. Ostomy Wound Manage 2002; 48: 52-60.
14. Bayat M, Jalili MR, Razavie N, et al. Effects of low-level laser therapy on mast cell number and degranulation in thirddegree burns of rats. J Rehabil Res Develop 2008; 45: 931-8.

15. Barbosa Pinheiro AL, Cavalcanti de Sousa AP, Matos Paraguassu G, et al. Laser and LED phototherapies on angiogenesis. Lasers Med Sci 2013; 28: 981-7. 